\title{
The Distance between the Lower Edge of the Xiphisternum and the Center of the Umbilicus as an Indicator of Abdominal Obesity and Cardiovascular Disease Risk
}

\author{
Prasad Katulanda ${ }^{a, b} \quad$ Mallika Arachchige Ranil Jayawardena ${ }^{a} \quad$ Mohamed Hussain Rezvi Sheriff $^{a}$ \\ David Richard Matthews ${ }^{\mathrm{b}}$
}

${ }^{a}$ Diabetes Research Unit, Department of Clinical Medicine, Faculty of Medicine, University of Colombo, Sri Lanka

${ }^{\mathrm{b}}$ Oxford Centre for Diabetes, Endocrinology and Metabolism, University of Oxford, UK

\section{Keywords}

Abdominal obesity - Anthropometry - CVD risk .

Xiphisternum · XUD

\section{Summary}

Objective: Abdominal obesity is considered an independent risk factor for cardiovascular disease (CVD). Aim: To compare the distance between the lower edge of $x i-$ phisternum and the center of the umbilicus (XUD) with other anthropometric measurements in predicting risk factors for CVD. Methods: A random sample of 4,485 (males 1,772, females 2,713 ) nationally representative noninstitutionalized adults aged $\geq 18$ years were included in the analysis. XUD, BMI, waist circumference (WC), waist-to-hip ratio (WHR), systolic and diastolic blood pressure were measured and fasting venous blood samples were collected to determine glucose, total cholesterol, low-density lipoprotein (LDL) cholesterol, high-density lipoprotein cholesterol and triacylglyceride levels. Oral glucose tolerance test (OGTT) was also preformed. Results: The mean XUD ( \pm SD) was $17.4 \pm 3.2 \mathrm{~cm}$ and $17.0 \pm$ $3.0 \mathrm{~cm}$ for males (M) and females (F) respectively ( $M$ vs. $F ; p<0.001)$. XUD had significant correlations with the cardiovascular risk factors analyzed as continuous variables ( $p<0.001$ ), except LDL cholesterol for women. However, the strengths of associations were weak for all associations $(<0.3)$. The correlations of BMI, WC and WHR with XUD were significant $(p<0.001)$. The area under the curve $(A \cup C)$ in the receiver operating characteristic (ROC) curve analysis for XUD in predicting the presence of two or more CVD risk factors was $0.62(95 \% \mathrm{Cl} 0.61-$ 0.64); the corresponding AUC values for $\mathrm{BMI}(0.71,95 \%$ $\mathrm{Cl}$ 0.69-0.72), WC (0.71 95\% Cl, 0.70-0.73), and WHR
(0.6795\% Cl, 0.65-0.68) were higher than that for XUD. Conclusion: The XUD showed significant correlations with the cardiovascular risk factors among Sri Lankan adults. However, in predicting CVD risk XUD was inferior to BMI, WC, and WHR.

\section{Introduction}

The prevalence of obesity has been increasing worldwide [1]. Abdominal obesity, even more than generalized adiposity, leads to insulin resistance and is postulated to play an important role in the development of diabetes and cardiovascular diseases (CVDs)[2]. The release of free fatty acids from visceral fat may increase dyslipidemia [3]. Moreover, the release of various signaling proteins and cytokines from abdominal fat tissue may also contribute to proinflammatory states and an unfavorable metabolic profile [4]. Various studies have shown that excess abdominal fat is associated with CVDs [3], diabetes [5] and an increased overall mortality risk [6]. Anthropometric parameters are widely used as surrogate markers of excess body fat. BMI may not be a good method to measure abdominal adiposity [7]. However, waist circumference (WC) or waist-to-hip ratio (WHR) has been shown to be not better than BMI in identifying abdominal adiposity-associated insulin resistance $[8,9]$. Some studies [10] showed that the sagittal abdominal diameter (SAD) is superior to other anthropometric measures to assess the abdominal obesity-associated disease risk, but other studies [11] did not. In addition, each of the anthropometric measures is associated with specific shortcomings.

\section{KARGER}

Fax +497614520714

Information@Karger.de

www.karger.com
(C) 2010 S. Karger GmbH, Freiburg

www.karger.com/ofa 
Table 1. Subjects characteristics

\begin{tabular}{lccc}
\hline Characteristics & $\begin{array}{l}\text { Male } \\
(\mathrm{n}=1,772)\end{array}$ & $\begin{array}{l}\text { Female } \\
(\mathrm{n}=2,713)\end{array}$ & p value* \\
\hline Age, years (SD) & $46.3(15.8)$ & $46.0(14.6)$ & 0.51 \\
XUD, cm (SD) & $17.4(3.2)$ & $17.0(3.0)$ & $<0.001$ \\
BMI, kg/m (SD) & $21.1(3.7)$ & $22.8(4.5)$ & $<0.001$ \\
WC, cm (SD) & $78.1(11.0)$ & $76.7(12.1)$ & $<0.001$ \\
WHR (SD) & $0.89(0.07)$ & $0.85(0.08)$ & $<0.001$ \\
SBP, mm Hg (SD) & $128.9(19.4)$ & $125.9(19.9)$ & $<0.001$ \\
DBP, mm Hg (SD) & $75.2(11.6)$ & $75.4(11.1)$ & 0.575 \\
FBG, mmol/l (SD) & $5.04(1.53)$ & $5.01(1.59)$ & 0.513 \\
2-hour plasma glucose, mmol/l (SD) & $5.68(2.85)$ & $6.33(2.79)$ & $<0.001$ \\
Total cholesterol, mmol/l (SD) & $5.2(1.1)$ & $5.4(1.1)$ & $<0.001$ \\
LDLC, mmol/l (SD) & $3.4(1.0)$ & $3.6(1.0)$ & $<0.001$ \\
HDLC, mmol/l (SD) & $1.16(0.27)$ & $1.25(0.27)$ & $<0.001$ \\
TAG, mmol/l (SD) & $1.50(0.83)$ & $1.29(0.69)$ & $<0.001$ \\
\hline
\end{tabular}

$\mathrm{SBP}=$ Systolic blood pressure $\mathrm{DBP}=$ diastolic blood pressure $; \mathrm{TAG}=$ triglycerides.

*t-test for equality of means between males and females.

Distances between the lower border of xiphisternum and the center of the umbilicus (XUD) has been used to develop tools for neonatal reconstruction surgeries [12, 13]. We hypothesized that XUD would be a valuable anthropometric measure to estimate abdominal adiposity and associated cardiovascular risk factors. The aim of this study was to evaluate the XUD as a marker of abdominal obesity and to compare its predictability of CVD risk with other conventional anthropometric indices.

\section{Material and Methods}

\section{Subjects}

This study was designed as a large cross-sectional study and conducted between August 2005 and September 2006. A total of 5,000 adults were randomly selected from the seven out of the nine provinces in Sri Lanka. A multi-stage random-cluster sampling method was used to select a nationally representative sample of noninstitutionalized adults aged $\geq 18$ years. The ethical approval for the study was obtained from the Ethical Review Committee of the University of Colombo, Sri Lanka. Written informed consent was obtained from each participant before data collection. Details of the study design has been published elsewhere [14].

\section{Measurements}

Weight and height were measured with the subjects wearing indoor light clothing without shoes; then BMI was calculated as weight $(\mathrm{kg}) /$ height $^{2}$ $\left(\mathrm{m}^{2}\right)$. WC was measured at the level midway between the lowest rib margin and the iliac crest, and hip circumference was measured at the widest level over the greater trochanters. WHR was calculated as WC divided by hip circumference. Anthropometric measures were performed by trained nurses adhering to the WHO guidelines using calibrated equipment [15]. In a standing position, XUD was measured as distance between the lower border of xiphisternum and the center of umbilicus at the end of normal expiration by plastic flexible tape to the nearest $0.1 \mathrm{~cm}$.

Seated blood pressure was measured twice with Omron IA2 digital blood pressure monitors (Omron Healthcare, Singapore) while the subject was sitting. The averages of the 2 measurements were used for the analysis.
Overnight fasting venous blood samples were collected to measure cholesterol, triacylglycerides and glucose. Cholesterol and triglycerides were measured by an enzymatic colorimetric method in a RxDaytona ${ }^{\mathrm{TM}}$ chemical analyzer (Randox Laboratories LTD, Antrim, UK). Participants underwent a single 75 -g oral glucose tolerance test (OGTT).

\section{Statistical Methods}

The data were analyzed using SPSS version 14 (SPSS Inc., Chicago, IL, USA) and Stata/SE 10.0 (Stata Corporation, College Station, TX, USA) statistical software packages. Descriptive statistics given are number of observations, mean and standard deviation (SD). 'CVD risk factors' were calculated and comprised fasting glucose, OGTT, serum triglycerides, total cholesterol, low-density lipoprotein (LDL) cholesterol, high-density lipoprotein (HDL) cholesterol as well as systolic and diastolic blood pressure. The relation between the metabolic risk score and each of the four anthropometric variables was analyzed using Pearson's correlation coefficient. Area under the curve (AUC) in the receiver operating characteristic curve (ROC) analysis was calculated for the presence of two or more components of metabolic syndrome [16] (other than the obesity parameter) and XUD as well as other anthropometric indices as independent covariates to determine their suitability in predicting the CVD risk of the participants.

\section{Results}

A total of 4,485 subjects completed the study (response rate $90 \%)$. The mean XUD ( \pm SD) was $17.4 \pm 3.2 \mathrm{~cm}$ and $17.0 \pm 3.0$ $\mathrm{cm}$ for men and women, respectively $(\mathrm{p}<0.001)$. The mean WC was $78.1 \pm 11.0 \mathrm{~cm}$ for men and $76.7 \pm 12.1 \mathrm{~cm}$ for women, and the mean WHR was $0.89 \pm 0.07$ for men and $0.85 \pm 0.08$ for women. Men had a mean BMI of $21.1 \pm 3.7 \mathrm{~kg} / \mathrm{m}^{2}$ and women $22.8 \pm 4.5 \mathrm{~kg} / \mathrm{m}^{2}$. Clinical and biochemical characteristics of the study participants are presented in table 1 .

The three traditional anthropometric indices significantly correlated with all cardiovascular risk factors $(\mathrm{p}<0.001$ for all correlations). Except for LDL cholesterol for females $(\mathrm{p}=0.025)$, also XUD strongly correlated with the risk factors 
Table 2. Correlation coefficients between anthropometric measurements and CVD risk factors ${ }^{\mathrm{a}}$

\begin{tabular}{|c|c|c|c|c|c|c|c|c|}
\hline & \multicolumn{2}{|l|}{ XUD } & \multicolumn{2}{|l|}{ BMI } & \multicolumn{2}{|l|}{ WC } & \multicolumn{2}{|l|}{ WHR } \\
\hline & male & female & male & female & male & female & male & female \\
\hline Fasting plasma glucose & 0.20 & 0.10 & 0.19 & 0.13 & 0.23 & 0.14 & 0.20 & 0.12 \\
\hline 2-hour plasma glucose & 0.21 & 0.12 & 0.24 & 0.20 & 0.25 & 0.20 & 0.22 & 0.15 \\
\hline LDL cholesterol & 0.15 & $0.02^{\dagger}$ & 0.27 & 0.17 & 0.29 & 0.20 & 0.24 & 0.17 \\
\hline HDL cholesterol & -0.13 & -0.15 & -0.17 & -0.22 & -0.16 & -0.23 & -0.11 & -0.16 \\
\hline Triglycerides & 0.15 & 0.18 & 0.33 & 0.28 & 0.35 & 0.27 & 0.32 & 0.19 \\
\hline Total cholesterol & 0.19 & 0.05 & 0.30 & 0.18 & 0.32 & 0.17 & 0.27 & 0.11 \\
\hline Systolic blood pressure & 0.17 & 0.14 & 0.25 & 0.28 & 0.24 & 0.26 & 0.18 & 0.15 \\
\hline Diastolic blood pressure & 0.22 & 0.22 & 0.29 & 0.35 & 0.31 & 0.32 & 0.24 & 0.19 \\
\hline
\end{tabular}

${ }^{\mathrm{a} X U D}, \mathrm{BMI}, \mathrm{WC}$ and WHR were significantly associated with all cardiovascular risk factors $(\mathrm{p}<0.001)$ except for XUD and LDL cholesterol in females $\left({ }^{\dagger} \mathrm{p}=0.025\right)$.
Table 3. Correlation coefficient between XUD with BMI, WC and WHR

\begin{tabular}{llllllll}
\hline & Male & & & & \multicolumn{2}{l}{ Female } \\
\cline { 2 - 3 } \cline { 7 - 8 } & BMI & WC & WHR & & BMI & WC & WHR \\
\hline XUD & $0.61 *$ & $0.68^{*}$ & $0.44^{*}$ & & $0.55^{*}$ & $0.55^{*}$ & $0.32 *$ \\
\hline
\end{tabular}

$* \mathrm{p}<0.001$.

Table 4. ROC curves in predicting CVD risk*

\begin{tabular}{ll}
\hline Anthropometric indices & AUC in the ROC curve analysis \\
\hline XUD & $0.62(0.61-0.64)$ \\
BMI & $0.71(0.69-0.72)$ \\
WC & $0.71(0.70-0.73)$ \\
WHR & $0.67(0.65-0.68)$ \\
\hline
\end{tabular}

*Presence of two or more factors of metabolic syndrome (except obesity).

analyzed as continuous variables $(\mathrm{p}<0.001$ for all correlations). However, the strength of the association was lowest in XUD compared to other anthropometric measurts (table 2).

XUD correlated significantly $(\mathrm{p}<0.001)$ with other anthropometric measures. Particularly in men, BMI and WC had strong correlations with XUD. The strength of association between WHR and XUD was less compared to BMI and WC (table 3).

ROC curve analysis showed an AUC $>0.7$ for $\mathrm{WC}$ and BMI indices in both sexes $(\mathrm{p}<0.001)$. This indicated significant and satisfactory prediction of metabolic disease. However, XUD showed an AUC of $<0.7$ and was inferior to the other anthropometric indices (table 4).

\section{Discussion}

To our knowledge, this is the first study to examine XUD as an anthropometric index to evaluate the obesity-associated cardiovascular risk profile. Required landmarks are quite easy to observe compared to WC and WHR measurements; therefore, inter- and intraobserver variations should be low. Moreover, only minimum of removal of outer clothes is needed, so this would be much preferable in some cultural settings compared to WC. No calculation is necessary to interpret results. Compared to BMI, WC and WHR, XUD is a tool easier to use in both clinical and community settings. XUD is strongly correlated with other indices, in particular BMI and WC. XUD would be an alternative anthropometric tool to assess obesity and CVD risk.

Among both men and women in this study, XUD was found to have significant correlations with risk factors for CVD. Enlarged fatty liver [17] and omental fat [18] are known risk factors for altered metabolic profile. These factors may be partially responsible for distension of upper abdomen (which is measured by XUD) in high-risk groups.

However, compared with the other indices XUD showed the weakest correlation with cardiovascular risk factors and thus was less suitable than the other indices in predicting obesity-related CVD. Therefore, it is difficult to justify usage of XUD over other anthropometric measurement in Sri Lankan adult population.

Our study group is primarily a South Asian (Sri Lankan) population. It is widely accepted that this people have other metabolic profiles than Caucasians [19]. Although, XUD is not superior to other anthropometric measures in South Asian ethnicity, the applicability of this index to non-South Asian groups needs to be investigated. Another limitation of XUD is that midline abdominal hernias may interfere with its measurement. Obesity on the other hand is a predisposing factor for midline hernias [20].

In summary, XUD was shown to be associated with obesity-associated risk factors for CVD. However, in Sri Lankan adults its association with cardiovascular risk factors was weaker than that of other anthropometric indices such as BMI, WC and WHR.

\section{Disclosure}

The authors declared no conflict of interest. 


\section{References}

1 James PT, Rachel L, Eleni K, Maryam S: The worldwide obesity epidemic. Obes Res 2001;9: S228-233.

2 Kahn BB, Flier JS: Obesity and insulin resistance. J Clin Invest 2000;106:473-481.

3 Després JP, Lemieux S, Lamarche B, Prud'homme D, Moorjani S, Brun LD, Gagné C, Lupien PJ The insulin resistance-dyslipidemic syndrome: contribution of visceral obesity and therapeutic implications. Int J Obes Relat Metab Disord 1995; 19(suppl):S76-86.

4 Jazet IM, Pijl H, Meinders AE: Adipose tissue as an endocrine organ: impact on insulin resistance. Neth J Med 2003;61:194-212.

5 Hayashi T, Boyko EJ, Leonetti DL, McNeely MJ, Newell-Morris L, Kahn SE: Visceral adiposity and the risk of impaired glucose tolerance: a prospective study among Japanese Americans. Diab. Care 2003;26:650-655.

6 Kuk JL, Katzmarzyk PT, Nichaman MZ, Church TS, Blair SN, Ross R: Visceral fat is an independent predictor of all cause mortality in men, Obesity 2006;14:336-341.

7 Deurenberg P, Yap M, van Staveren WA: Body mass index and percent body fat: a meta analysis among different ethnic groups. Int J Obes Relat Metab Disord 1998;22:1164-1171.

8 McLaughlin T, Abbasi F, Cheal K, Chu J, Lamendola C, Reaven G: Use of metabolic markers to identify overweight individuals who are insulin resistant. Ann Intern Med 2003;139:802-809.
9 Ferrannini E, Natali A, Bell P, Cavallo-Perin P, Lalic N, Mingrone G: Insulin resistance and hypersecretion in obesity: European Group for the Study of Insulin Resistance (EGIR). J Clin Invest 1997;100:1166-1173.

10 Ohrvall M, Berglund L, Vessby B: Sagittal abdominal diameter compared with other anthropometric measurements in relation to cardiovascular risk. Int J Obes 2000;24:497-501.

11 Mukuddem-Petersen J, Snijder MB, Dam RM, Dekker JM, Bouter JM, Stehouwer CDA, Heine RJ, Nijpels G, Seidell JC: Sagittal abdominal diameter: no advantage compared with other anthropometric measures as a correlate of components of the metabolic syndrome in elderly from the Hoorn Study. Am J Clin Nutr 2006;84:995-1002.

12 Davari $\mathrm{H}$, Nazem M: The normal position of umbilicus in the newborn: an aid to improve cosmetic result in exomphalos major. J Res Med Sci 2004;1:34-38.

13 Abhyankar SV, Rajguru AG, Patil PA: Anatomical localization of the umbilicus: an Indian study. Plast Reconstr Surg 2006;117:1153-1157.

14 Katulanda P, Constantine GR, Mahesh JG, Sheriff R, Seneviratne RDA, Wijeratne S, Wijesuriya $\mathrm{M}$, McCarthy MI, Adler AI, Matthews RD: Prevalence and projections of diabetes and prediabetes in adults in Sri Lanka - Sri Lanka Diabetes, Cardiovascular Study (SLDCS). Diabet Med 2008;25:1062-1069.
15 World Health Organization: Physical status: the use and interpretation of anthropometry. Report of a WHO Expert Committee. Geneva, World Health Organization, 1995, WHO Technical Report Series, No 854, pp 424-438.

16 Alberti KGMM, Zimmet P, Shaw J: The IDF Epidemiology Task Force Consensus Group: The metabolic syndrome: a new worldwide definition. Lancet 2005;366:1059-1062.

17 Marceau P, Biron S, Hould FS, Marceau S, Simard S, Thung SN, Kral JG: Liver pathology and the metabolic syndrome $\mathrm{X}$ in severe obesity. J Clin Endocrinol Metab 1999;84:1513-1517.

18 Thorne A, Lonnqvist F, Apelman J, Hellers G, Arner P: A pilot study of long-term effects of a novel obesity treatment: omentectomy in connection with adjustable gastric banding. Int $\mathrm{J}$ Obes Relat Metab Disord 2002;26:193-199.

19 Mathews R, Zachariah R: Coronary heart disease in south asian immigrants. J Transcult Nurs 2008; 19:292-299.

20 Hodgson TJ, Collins MC: Anterior abdominal wall hernias: diagnosis by ultrasound and tangential tangential radiographs. Clin Radiol 1991;44: 185-188. 